\title{
Solutions to impulsive integrodifferential evolution equations under a noncompact evolution system
}

\author{
Shaochun $\mathrm{Ji}^{i^{*}}$ and Min Wang ${ }^{2}$
}

"Correspondence:

jiscmath@163.com

${ }^{1}$ Faculty of Mathematics and

Physics, Huaiyin Institute of

Technology, Huaian, 223003,

P.R. China

Full list of author information is

available at the end of the article

\begin{abstract}
In this paper, we study the existence of mild solutions to impulsive integrodifferential evolution equations in Banach spaces. Based on a measure of noncompactness and important properties of semicompact sets, new existence results are obtained. Here the evolution system is only supposed to be strongly continuous, without any compact or equicontinuous assumptions. Some applications are given to illustrate the effectiveness of our results.
\end{abstract}

MSC: $34 \mathrm{~A} 37 ; 34 \mathrm{G} 20 ; 47 \mathrm{D} 60$

Keywords: impulsive conditions; nonlocal conditions; integrodifferential equation; mild solution; measure of noncompactness

\section{Introduction}

This paper is concerned with integrodifferential evolution equations with impulsive conditions and nonlocal conditions:

$$
\left\{\begin{array}{l}
u^{\prime}(t)=A(t) u(t)+f\left(t, u(t), \int_{0}^{t} h(t, s, u(s)) \mathrm{d} s\right), \\
\quad t \in J=[0, b], t \neq t_{i}, i=1,2, \ldots, p, \\
\Delta u\left(t_{i}\right)=u\left(t_{i}^{+}\right)-u\left(t_{i}^{-}\right)=I_{i}\left(u\left(t_{i}\right)\right), \quad i=1,2, \ldots, p, \\
u(0)=g(u)+u_{0},
\end{array}\right.
$$

where $A(t)$ is a family of linear operators which generates an evolution system $\{U(t, s): 0 \leq$ $s \leq t \leq b\}$. The state variable $u(\cdot)$ takes values in the real Banach space $X$. The operators $h: T \times X \rightarrow X, f: J \times X \times X \rightarrow X$ are continuous, $T=\{(t, s), 0 \leq s \leq t \leq b\} . I_{i}: X \rightarrow X$, $i=1,2, \ldots, p$, are impulsive functions, $0<t_{1}<t_{2}<\cdots<t_{p}<t_{p+1}=b . \Delta u\left(t_{i}\right)$ is the jump of a function $u$ at $t_{i}, u\left(t_{i}^{-}\right), u\left(t_{i}^{+}\right)$denote the left and the right limit of $u$ at $t_{i}$, respectively. $g: P C([0, b] ; X) \rightarrow X$ is an appropriate continuous function to be specified later.

The impulsive differential systems can be used to model processes which are subject to short perturbations whose duration can be negligible in comparison with the duration of the process, such as the dynamics of populations subject to abrupt changes. For more details of this theory and its applications, we refer to the monographs of Lakshmikantham et al. [1] and Benchohra et al. [2], and to [3-5] and the references therein. In [6], Fan and Li used the techniques of approximate solutions and fixed points to get the mild solutions

(c) $2015 \mathrm{Ji}$ and Wang. This article is distributed under the terms of the Creative Commons Attribution 4.0 International License (http://creativecommons.org/licenses/by/4.0/), which permits unrestricted use, distribution, and reproduction in any medium, provided you give appropriate credit to the original author(s) and the source, provide a link to the Creative Commons license, and indicate if changes were made. 
of nonlocal impulsive differential equation

$$
\begin{aligned}
& u^{\prime}(t)=A u(t)+f(t, u(t)), \quad t \in[0, b], t \neq t_{i}, \\
& \Delta u\left(t_{i}\right)=u\left(t_{i}^{+}\right)-u\left(t_{i}^{-}\right)=I_{i}\left(u\left(t_{i}\right)\right), \quad i=1,2, \ldots, p, \\
& u(0)=g(u),
\end{aligned}
$$

where $A$ generates a compact semigroup $T(t)$, the impulsive functions $I_{i}$ are not compact. Abada et al. [7] studied the existence of integral solutions and extremal integral solutions for some nondensely defined impulsive semilinear functional differential inclusions in separable Banach spaces. Ji et al. [8, 9] studied the existence and controllability of solutions to impulsive differential system when the semigroup is equicontinuous.

On the other hand, the abstract nonlocal initial problem was initiated by Byszewski and Lakshmikantham [10, 11], where the existence and uniqueness of solutions to semilinear nonlocal differential equations were discussed. The importance of the problem consists in the fact that it is more general and has a better effect than the classical initial conditions $u(0)=u_{0}$. Therefore the nonlocal Cauchy problem has been studied extensively under various conditions on $A(t)$ and $f, g$, by several authors [12-16]. Ntouyas and Tsamatos [14] studied the nonlocal semilinear differential equations with compact conditions. Xue [15] discussed the semilinear nonlocal differential equations when the semigroup $T(t)$ generated by the coefficient operator is compact and the nonlocal function $g$ is not compact. Some classes of integrodifferential equations with nonlocal conditions have been investigated by Balachandran et al. [17, 18]. Wang and Wei [19] and Machado et al. [20] discussed the existence of a class of impulsive integrodifferential evolution equations, where the evolution system is supposed to be equicontinuous.

In the above work, we find that the compactness of the evolution system plays a key role in this type of impulsive nonlocal Cauchy problem. However, sometimes it is difficult to satisfy. For example, let $X=L^{2}(-\infty,+\infty)$. The ordinary differential operator $A=\mathrm{d} / \mathrm{d} x$ with $D(A)=H^{1}(-\infty,+\infty)$, generates a semigroup $T(t)$ defined by $T(t) u(s)=u(t+s)$, for every $u \in X$. The $C_{0}$-semigroup $T(t)$ is not compact on $X$.

Recently, by using a new two-component measure of noncompactness, Benchohra and Ziane [21] proved the existence of mild solutions for a class of impulsive semilinear evolution differential inclusions with state-dependent delay when $A(t)$ generates a strongly continuous evolution operator. It is an interesting result. In this paper we explain that the existence results of differential systems under a noncompact evolution system can also be obtained via the classical Hausdorff measure of noncompactness. By applying the property of semicompact sets (see Lemma 2.5), we discuss the existence of mild solutions to (1.1) without the compactness of evolution system $U(t, s)$, even its equicontinuity. This is one motivation of the present work. Note that the assumption on the evolution system here is weaker than that in $[9,19,20]$, and no more conditions are added. The Banach space here is nonseparable. Another motivation of the present work is the exact controllability problem of the differential system. Our method can also be applied to an impulsive control system and can deal with the technical error on the exact controllability of differential system caused by the compactness of the evolution system (see Remark 4.2).

The paper is organized as follows. In Section 2 we recall some preliminary facts that we need in the sequel. In Section 3 we prove our results when the evolution system $U(t, s)$ is strongly continuous. Some applications of our results are given in Section 4. 


\section{Preliminaries}

Let $(X,\|\cdot\|)$ be a real Banach space. We denote by $C([0, b] ; X)$ the space of $X$-valued continuous functions on $[0, b]$ with the norm $\|x\|=\sup \{\|x(t)\|, t \in[0, b]\}$ and by $L^{1}([0, b] ; X)$ the space of $X$-valued Bochner integrable functions on $[0, b]$ with the norm $\|f\|_{L^{1}}=$ $\int_{0}^{b}\|f(t)\| \mathrm{d} t$.

For the sake of simplicity, we put $J=[0, b] ; J_{0}=\left[0, t_{1}\right] ; J_{i}=\left(t_{i}, t_{i+1}\right], i=1, \ldots, p$. In order to define the mild solution of problem (1.1), we introduce the set $P C([0, b] ; X)=\{u:[0, b] \rightarrow$ $X$ such that $u(\cdot)$ is continuous except for a finite number of points $t_{i}$, at which $u\left(t_{i}^{+}\right), u\left(t_{i}^{-}\right)$ exist and $\left.u\left(t_{i}\right)=u\left(t_{i}^{-}\right), i=1, \ldots, p\right\}$. It is easy to verify that $P C([0, b] ; X)$ is a Banach space with the norm $\|u\|_{P C}=\sup \{\|u(t)\|, t \in[0, b]\}$.

Now we recall the Hausdorff measure of noncompactness (in short MNC) $\beta(\cdot)$ defined by

$$
\beta(B)=\inf \{\varepsilon>0 ; B \text { has a finite } \varepsilon \text {-net in } X\}
$$

for each bounded subset $B$ in Banach space $X$. We recall the following properties of the Hausdorff measure of noncompactness $\beta$.

Lemma 2.1 ([22]) Let $X$ be a real Banach space and $B, C \subseteq X$ be bounded. Then the following properties are satisfied:

(1) $B$ is relatively compact if and only if $\beta(B)=0$;

(2) $\beta(B)=\beta(\bar{B})=\beta(\operatorname{conv} B)$, where $\bar{B}$ and $\operatorname{conv} B$ mean the closure and convex hull of $B$, respectively;

(3) $\beta(B) \leq \beta(C)$ when $B \subseteq C$;

(4) $\beta(B+C) \leq \beta(B)+\beta(C)$, where $B+C=\{x+y: x \in B, y \in C\}$;

(5) $\beta(B \cup C) \leq \max \{\beta(B), \beta(C)\}$;

(6) $\beta(\lambda B) \leq|\lambda| \beta(B)$ for any $\lambda \in \mathbb{R}$;

(7) if the map $Q: D(Q) \subseteq X \rightarrow Z$ is Lipschitz continuous with constant $k$, then $\beta_{Z}(Q B) \leq k \beta(B)$ for any bounded subset $B \subseteq D(Q)$, where $Z$ is a Banach space.

A two parameter family of bounded linear operators $\{U(t, s), 0 \leq s \leq t \leq b\}$ on $X$ is called an evolution system if the following two conditions are satisfied:

(i) $U(s, s)=I, U(t, r) U(r, s)=U(t, s)$ for $0 \leq s \leq r \leq t \leq b$;

(ii) $(t, s) \rightarrow U(t, s)$ is strongly continuous for $0 \leq s \leq t \leq b$.

In a natural way, we can consider the respective evolution operator $U: J \times J \rightarrow L(X)$, where $L(X)$ is the space of all bounded linear operators in $X$. Since the evolution system $U(t, s)$ is strongly continuous on the compact set $J \times J$, there exists $M>0$ such that $\|U(t, s)\| \leq M$ for any $(t, s) \in J \times J$. More details as regards this evolution system can be found in Pazy [23].

Definition 2.2 A function $u \in P C([0, b] ; X)$ is said to be a mild solution of (1.1) if

$$
u(t)=U(t, 0) u(0)+\int_{0}^{t} U(t, s) f\left(s, u(s), \int_{0}^{s} h(s, \tau, u(\tau)) \mathrm{d} \tau\right) \mathrm{d} s+\sum_{0<t_{i}<t} U\left(t, t_{i}\right) I_{i}\left(u\left(t_{i}\right)\right)
$$

for all $t \in[0, b]$, where $u(0)=g(u)+u_{0}$. 
Definition 2.3 A countable set $\left\{f_{n}\right\}_{n=1}^{+\infty} \subset L^{1}([0, b] ; X)$ is said to be semicompact if

- the sequence $\left\{f_{n}(t)\right\}_{n=1}^{+\infty}$ is relatively compact in $X$ for a.a. $t \in[0, b]$;

- there is a function $\mu \in L^{1}\left([0, b] ; \mathbb{R}^{+}\right)$satisfying $\sup _{n \geq 1}\left\|f_{n}(t)\right\| \leq \mu(t)$ for a.e. $t \in[0, b]$.

Lemma 2.4 ([24], Theorem 4.2.2) Let $\left\{f_{n}\right\}_{n=1}^{+\infty}$ be a sequence of functions in $L^{1}([0, b] ; X)$. Assume that there exist $\mu, \eta \in L^{1}\left([0, b] ; R^{+}\right)$satisfying

$$
\sup _{n \geq 1}\left\|f_{n}(t)\right\| \leq \mu(t) \quad \text { and } \quad \beta\left(\left\{f_{n}(t)\right\}_{n=1}^{+\infty}\right) \leq \eta(t) \quad \text { a.e. } t \in[0, b]
$$

Then for all $t \in[0, b]$, we have

$$
\beta\left(\left\{\int_{0}^{t} U(t, s) f_{n}(s) \mathrm{d} s: n \geq 1\right\}\right) \leq 2 M \int_{0}^{t} \eta(s) \mathrm{d} s
$$

The following lemma can be found in Theorem 2 of [25] and Theorem 5.1.1 of [24].

Lemma 2.5 Let $(G f)(t)=\int_{0}^{t} U(t, s) f(s) \mathrm{d}$. If $\left\{f_{n}\right\}_{n=1}^{+\infty} \subset L^{1}([0, b] ; X)$ is semicompact, then the set $\left\{G f_{n}\right\}_{n=1}^{+\infty}$ is relatively compact in $C([0, b] ; X)$ and, moreover, if $f_{n} \rightarrow f_{0}$, then for all $t \in$ $[0, b],\left(G f_{n}\right)(t) \rightarrow(G f)(t)$ as $n \rightarrow+\infty$.

Lemma 2.6 ([26]) Let $D$ be a bounded set of $X$. Then for any $\varepsilon>0$, there exists a sequence $\left\{u_{k}\right\}_{k=1}^{\infty} \subset D$ such that

$$
\beta(D) \leq 2 \beta\left(\left\{u_{k}\right\}_{k=1}^{\infty}\right)+\varepsilon
$$

Lemma 2.7 ([9]) If $W \subseteq P C([0, b] ; X)$ is bounded, then $\beta(W(t)) \leq \beta(W)$ for all $t \in[0, b]$, where $W(t)=\{u(t) ; u \in W\} \subseteq X$. Furthermore, suppose the following conditions are satisfied:

(1) $W$ is equicontinuous on $J_{0}=\left[0, t_{1}\right]$ and each $J_{i}=\left(t_{i}, t_{i+1}\right], i=1, \ldots, p$;

(2) $W$ is equicontinuous at $t=t_{i}^{+}, i=1, \ldots, p$.

Then $\sup _{t \in[0, b]} \beta(W(t))=\beta(W)$.

Throughout this paper, we denote $M=\sup \{\|U(t, s)\|:(t, s) \in J \times J\}, W_{r}=\{u \in P C([0, b]$; $X):\|u(t)\| \leq r, \forall t \in[0, b]\}$. Without loss of generality, we let $u_{0}=0$.

\section{Main results}

First we give the following hypotheses:

$\left(\mathrm{H}_{1}\right) A(t)$ is a family of linear (not necessarily bounded) operators and $A(t): D(A) \rightarrow X$ generates a strongly continuous evolution system $\{U(t, s): 0 \leq s \leq t \leq b\}, D(A)$ not depending on $t$ and a dense subset of $X$ (see [23]).

$\left(\mathrm{H}_{2}\right) g: P C([0, b] ; X) \rightarrow X$ is continuous and compact.

$\left(\mathrm{H}_{3}\right) I_{i}: X \rightarrow X$ is continuous and compact for each $i=1,2, \ldots, p$.

$\left(\mathrm{H}_{4}\right)$ The function $f:[0, b] \times X \times X \rightarrow X$ satisfies the following:

(1) For a.e. $t \in[0, b]$, the function $f(t, \cdot, \cdot): X \times X \rightarrow X$ is continuous and for all $x, y \in X \times X$, the function $f(\cdot, x, y):[0, b] \rightarrow X$ is strongly measurable. 
(2) There exists a function $\theta \in L^{1}\left(J ; \mathbb{R}^{+}\right)$such that

$$
\|f(t, x, y)\| \leq \theta(t)(\|x\|+\|y\|)
$$

for a.e. $t \in[0, b]$ and all $x, y \in X$.

(3) There exists a function $l \in L^{1}\left(J ; R^{+}\right)$such that for every bounded set $A, B \subset X$,

$$
\beta(f(t, A, B)) \leq l(t)[\beta(A)+\beta(B)]
$$

for a.e. $t \in[0, b]$.

$\left(\mathrm{H}_{5}\right)$ The function $h: T \times X \rightarrow X$ satisfies the following:

(1) For a.e. $(t, s) \in T$, the function $h(t, s, \cdot): X \rightarrow X$ is continuous and for all $x \in X$, the function $h(\cdot, \cdot, x): T \rightarrow X$ is strongly measurable.

(2) There exists a function $m \in L^{1}\left(T ; \mathbb{R}^{+}\right)$such that

$$
\|h(t, s, x)\| \leq m(t, s)\|x\| .
$$

Let us take $m^{*}=\max _{(t, s) \in T} \int_{0}^{t} m(t, s) \mathrm{d} s$.

(3) There exist functions $\zeta_{1}, \zeta_{2} \in L^{1}\left(J ; \mathbb{R}^{+}\right)$such that

$$
\beta(h(t, s, D)) \leq \zeta_{1}(t) \zeta_{2}(s) \beta(D)
$$

for a.e. $(t, s) \in T, D \subset X$ a bounded set.

Now, we give the existence result under the above hypotheses.

Theorem 3.1 Assume that the hypotheses $\left(\mathrm{H}_{1}\right)-\left(\mathrm{H}_{5}\right)$ are satisfied, then the nonlocal impulsive problem (1.1) has at least one mild solution on $[0, b]$, provided that

$$
M\left[\sup _{u \in W_{r}}\|g(u)\|+r\|\theta\|_{L^{1}}+m^{*} r\|\theta\|_{L^{1}}+\sup _{u \in W_{r}} \sum_{i=1}^{p}\left\|I_{i}\left(u\left(t_{i}\right)\right)\right\|\right] \leq r .
$$

Proof Put the map $K: P C([0, b] ; X) \rightarrow P C([0, b] ; X)$ defined by

$$
(K u)(t)=\left(K_{1} u\right)(t)+\left(K_{2} u\right)(t)
$$

with

$$
\begin{aligned}
& \left(K_{1} u\right)(t)=U(t, 0) g(u)+\int_{0}^{t} U(t, s) f\left(s, u(s), \int_{0}^{s} h(s, \tau, u(\tau)) \mathrm{d} \tau\right) \mathrm{d} s \\
& \left(K_{2} u\right)(t)=\sum_{0<t_{i}<t} U\left(t, t_{i}\right) I_{i}\left(u\left(t_{i}\right)\right)
\end{aligned}
$$

for all $t \in[0, b]$.

It is easy to see that the fixed point of $K$ is the mild solution of nonlocal impulsive problem (1.1). Subsequently, we will prove that $K$ has a fixed point by using the Schauder fixed point theorem. 
From our hypotheses, we see that $K$ is continuous on $P C(J ; X)$. For this purpose, we assume that $u_{n} \rightarrow u$ in $P C(J ; X)$. By hypotheses $\left(\mathrm{H}_{4}\right)$ and $\left(\mathrm{H}_{5}\right)$, we have

$$
f\left(s, u_{n}(s), \int_{0}^{s} h\left(s, \tau, u_{n}(\tau)\right) \mathrm{d} \tau\right) \rightarrow f\left(s, u(s), \int_{0}^{s} h(s, \tau, u(\tau)) \mathrm{d} \tau\right), \quad n \rightarrow+\infty
$$

for $s \in[0, b]$.

Then from the continuity of $g, I_{i}$ and the dominated convergence theorem, we have

$$
\begin{aligned}
\left\|K u_{n}-K u\right\|_{P C} \leq & M\left\|g\left(u_{n}\right)-g(u)\right\| \\
& +M \int_{0}^{b} \| f\left(s, u_{n}(s), \int_{0}^{s} h\left(s, \tau, u_{n}(\tau)\right) \mathrm{d} \tau\right) \\
& -f\left(s, u(s), \int_{0}^{s} h(s, \tau, u(\tau)) \mathrm{d} \tau\right) \| \mathrm{d} s \\
& +\sum_{i=1}^{p} M\left\|I_{i}\left(u_{n}\left(t_{i}\right)\right)-I_{i}\left(u\left(t_{i}\right)\right)\right\| \rightarrow 0, \quad \text { as } n \rightarrow+\infty,
\end{aligned}
$$

i.e., $K$ is continuous.

We denote by $W_{0}=\{u \in P C([0, b] ; X):\|u(t)\| \leq r$ for all $t \in[0, b]\}$. Then $W_{0} \subset P C([0, b]$; $X)$ is bounded and convex.

Define $W_{1}=\overline{\mathrm{co}}\left\{K\left(W_{0}\right), u_{0}\right\}$, where $\overline{\text { co }}$ means the closure of the convex hull in $P C([0, b]$; $X), u_{0} \in W_{0}$. For any $u \in K\left(W_{0}\right)$, we know that

$$
\begin{aligned}
\|u(t)\| \leq & M\left[\|g(u)\|+\int_{0}^{b} \theta(s)\left(\|u(s)\|+\int_{0}^{s}\|h(s, \tau, u(\tau))\| \mathrm{d} \tau\right) \mathrm{d} s\right] \\
& +M \sum_{i=1}^{p}\left\|I_{i}\left(u\left(t_{i}\right)\right)\right\| \\
\leq & M\left[\sup _{u \in W_{r}}\|g(u)\|+r\|\theta\|_{L^{1}}+m^{*} r\|\theta\|_{L^{1}}+\sup _{u \in W_{r}} \sum_{i=1}^{p}\left\|I_{i}\left(u\left(t_{i}\right)\right)\right\|\right]
\end{aligned}
$$

for $t \in[0, b]$. From (3.1), it follows that $W_{1} \subset W_{0}$.

We define

$$
W_{n+1}=\overline{\mathrm{co}}\left\{K\left(W_{n}\right), u_{0}\right\} \text { for } n=1,2, \ldots
$$

It is easy to show that $\left\{W_{n}\right\}_{n=1}^{\infty}$ is a decreasing sequence of bounded, closed, convex subsets in $P C([0, b] ; X)$. Then the set $W=\bigcap_{n=1}^{\infty} W_{n}$ is a nonempty bounded convex closed subset in $P C([0, b] ; X)$. Taking the limit in both sides of (3.2), we have $W=\overline{c o}\left\{K(W), u_{0}\right\}$.

Now we shall prove that $W$ is relatively compact in $P C([0, b] ; X)$.

For $n \geq 1$ and $t \in[0, b], W_{n}(t)$ and $K\left(W_{n}(t)\right)$ are bounded subsets of $X$. Hence, from Lemma 2.6, for any $\varepsilon>0$ and each $t \in[0, b]$, there is a sequence $\left\{u_{k}^{(n)}\right\}_{k=1}^{\infty} \subset W_{n}$ such that

$$
\begin{aligned}
\beta\left(W_{n+1}(t)\right) & =\beta\left(\overline{\operatorname{co}}\left\{\left(K W_{n}\right)(t), u_{0}(t)\right\}\right)=\beta\left(K W_{n}(t)\right) \\
& \leq 2 \beta\left(\left\{\left(K u_{k}^{(n)}\right)(t)\right\}_{k=1}^{\infty}\right)+\varepsilon
\end{aligned}
$$




$$
\begin{aligned}
\leq & 2 \beta\left(U(t, 0) g\left(\left\{u_{k}^{(n)}\right\}_{k=1}^{\infty}\right)\right) \\
& +2 \beta\left(\int_{0}^{t} U(t, s) f\left(s,\left\{u_{k}^{(n)}(s)\right\}_{k=1}^{\infty},\left\{\int_{0}^{s} h\left(s, \tau, u_{k}^{(n)}(\tau)\right) \mathrm{d} \tau\right\}_{k=1}^{\infty}\right) \mathrm{d} s\right) \\
& +2 \sum_{i=1}^{p} \beta\left(U\left(t, t_{i}\right) I_{i}\left(\left\{u_{k}^{(n)}\left(t_{i}\right)\right\}_{k=1}^{\infty}\right)\right)+\varepsilon .
\end{aligned}
$$

From the compactness of $g$ and $I_{i}$, we get

$$
\begin{aligned}
& \beta\left(U(t, 0) g\left(\left\{u_{k}^{(n)}\right\}_{k=1}^{\infty}\right)\right)=0, \\
& \beta\left(U\left(t, t_{i}\right) I_{i}\left(\left\{u_{k}^{(n)}\left(t_{i}\right)\right\}_{k=1}^{\infty}\right)\right)=0
\end{aligned}
$$

for each $i=1, \ldots, p$. Then by Lemma 2.4 and hypothesis $\left(\mathrm{H}_{4}\right)$, we have

$$
\begin{aligned}
\beta\left(W_{n+1}(t)\right) \leq & 2 \beta\left(\int_{0}^{t} U(t, s) f\left(s,\left\{u_{k}^{(n)}(s)\right\}_{k=1}^{\infty},\left\{\int_{0}^{s} h\left(s, \tau, u_{k}^{(n)}(\tau)\right) \mathrm{d} \tau\right\}_{k=1}^{\infty}\right) \mathrm{d} s\right)+\varepsilon \\
\leq & 4 M \int_{0}^{t} \beta\left(f\left(s,\left\{u_{k}^{(n)}(s)\right\}_{k=1}^{\infty},\left\{\int_{0}^{s} h\left(s, \tau, u_{k}^{(n)}(\tau)\right) \mathrm{d} \tau\right\}_{k=1}^{\infty}\right)\right) \mathrm{d} s+\varepsilon \\
\leq & 4 M \int_{0}^{t} l(s)\left[\beta\left(\left\{u_{k}^{(n)}(s)\right\}_{k=1}^{\infty}\right)+\beta\left(\left\{\int_{0}^{s} h\left(s, \tau, u_{k}^{(n)}(\tau)\right) \mathrm{d} \tau\right\}_{k=1}^{\infty}\right)\right] \mathrm{d} s+\varepsilon \\
\leq & 4 M\left[\int_{0}^{t} l(s) \beta\left(\left\{u_{k}^{(n)}(s)\right\}_{k=1}^{\infty}\right) \mathrm{d} s\right. \\
& \left.+2 \int_{0}^{t} l(s)\left(\int_{0}^{s} \beta\left(\left\{h\left(s, \tau, u_{k}^{(n)}(\tau)\right)\right\}_{k=1}^{\infty}\right) \mathrm{d} \tau\right) \mathrm{d} s\right]+\varepsilon \\
\leq & 4 M\left[\int_{0}^{t} l(s) \beta\left(W_{n}(s)\right) \mathrm{d} s+2 \int_{0}^{t} l(s)\left(\int_{0}^{s} \beta\left(h\left(s, \tau, W_{n}(\tau)\right)\right) \mathrm{d} \tau\right) \mathrm{d} s\right]+\varepsilon \\
\leq & 4 M\left[\int_{0}^{t} l(s) \beta\left(W_{n}(s)\right) \mathrm{d} s+2 \int_{0}^{t} \int_{0}^{s} l(s) \zeta_{1}(s) \zeta_{2}(\tau) \beta\left(W_{n}(\tau)\right) \mathrm{d} \tau \mathrm{d} s\right]+\varepsilon \\
\leq & 4 M\left[\int_{0}^{t} l(s) \beta\left(W_{n}(s)\right) \mathrm{d} s+2 \int_{0}^{t} \zeta_{2}(\tau) \beta\left(W_{n}(\tau)\right) \mathrm{d} \tau \cdot \int_{0}^{t} l(s) \zeta_{1}(s) \mathrm{d} s\right]+\varepsilon
\end{aligned}
$$

for each $t \in[0, b]$. We let $\gamma=\max _{t \in[0, b]} \int_{0}^{t} l(s) \zeta_{1}(s) \mathrm{d} s, k(s)=4 M l(s)+8 M \gamma \zeta_{2}(s)$. Since $\varepsilon>0$ is arbitrary, it follows from the above inequality that

$$
\beta\left(W_{n+1}(t)\right) \leq \int_{0}^{t} k(s) \beta\left(W_{n}(s)\right) \mathrm{d} s
$$

for each $t \in[0, b]$. As $W_{n}$ is decreasing for $n$, we define

$$
\eta(t)=\lim _{n \rightarrow \infty} \beta\left(W_{n}(t)\right)
$$

for each $t \in[0, b]$. Taking the limit in both sides of (3.3), we obtain

$$
\eta(t) \leq \int_{0}^{t} k(s) \eta(s) \mathrm{d} s \quad \text { for each } t \in[0, b] .
$$

Noticing that $\eta(\cdot) \in L^{1}\left([0, b] ; \mathbb{R}^{+}\right)$is not continuous on $[0, b]$, we cannot apply the classical Gronwall inequality to obtain $\eta(t)=0$ for $t \in[0, b]$. This is due to the fact that the conti- 
nuity of $\beta\left(W_{n}(t)\right)$ for $t \in[0, b]$ is based on the equicontinuity of $U(t, s)$, which is absent in our assumptions. Thus we look for a new way to consider the problem.

Set $\eta_{n}(t)=\beta\left(W_{n}(t)\right), \rho_{n+1}(t)=\int_{0}^{t} k(s) \eta_{n}(s) \mathrm{d} s$. As for each $t \in[0, b], \eta_{n}(t)$ is a decreasing sequence and $\eta(t)=\lim _{n \rightarrow \infty} \eta_{n}(t)$, we see that $\lim _{n \rightarrow \infty} \rho_{n+1}(t)$ exists (the proof is similar to the Levi lemma except for a trivial modification). Let $\rho(t)=\lim _{n \rightarrow \infty} \rho_{n+1}(t)$. Taking the limit in both sides of $\rho_{n+1}(t)=\int_{0}^{t} k(s) \eta_{n}(s) \mathrm{d} s$, we have

$$
\rho(t) \leq \int_{0}^{t} k(s) \eta(s) \mathrm{d} s
$$

$\rho$ is a continuous function. From (3.3), we have $\eta_{n}(t) \leq \int_{0}^{t} k(s) \eta_{n-1}(s) \mathrm{d} s=\rho_{n}(t)$. Taking the limit in both sides, we get

$$
\eta(t) \leq \rho(t)
$$

From inequalities (3.4) and (3.5), we derive that

$$
\rho(t) \leq \int_{0}^{t} k(s) \rho(s) \mathrm{d} s
$$

here $\rho(t)$ is continuous on $[0, b]$. Using the Gronwall inequality, we conclude that $\rho(t) \equiv 0$ on $[0, b]$. As $\eta(t) \leq \rho(t)$, it follows that $\eta(t) \equiv 0$ on $[0, b]$. That is,

$$
\eta(t)=\lim _{n \rightarrow \infty} \beta\left(W_{n}(t)\right)=0 \quad \text { for each } t \in[0, b] .
$$

As $W(t)=\bigcap_{n=1}^{\infty} W_{n}(t)=\lim _{n \rightarrow \infty} W_{n}(t)$, from (3.6), we have

$$
\beta(W(t))=0
$$

for each $t \in[0, b]$.

Subsequently, we shall prove that $\beta(K W)=0$. To this end, from Lemma 2.6, we see that, for any $\varepsilon>0$, there exists a sequence $\left\{y_{n}\right\}_{n=1}^{\infty} \subset K W$ such that

$$
\beta(K W) \leq 2 \beta\left(\left\{y_{n}\right\}_{n=1}^{\infty}\right)+\varepsilon .
$$

Then there exists $\left\{u_{n}\right\}_{n=1}^{\infty} \subset W$, such that

$$
y_{n}=K_{1}\left(u_{n}\right)+K_{2}\left(u_{n}\right) .
$$

Firstly, we show that $\left\{K_{1}\left(u_{n}\right)\right\}_{n=1}^{\infty} \subset C([0, b] ; X)$ is relatively compact, making full use of Lemma 2.5. From hypotheses $\left(\mathrm{H}_{4}\right)(3)$ and $\left(\mathrm{H}_{5}\right)(3)$, we get

$$
\begin{aligned}
& \beta\left(\left\{f\left(s, u_{n}(s), \int_{0}^{s} h\left(s, \tau, u_{n}(\tau)\right) \mathrm{d} \tau\right)\right\}_{n=1}^{\infty}\right) \\
& \quad \leq l(s)\left[\beta\left(\left\{u_{n}(s)\right\}_{n=1}^{\infty}\right)+\beta\left(\left\{\int_{0}^{s} h\left(s, \tau, u_{n}(\tau)\right) \mathrm{d} \tau\right\}_{n=1}^{\infty}\right)\right] \\
& \quad \leq l(s)\left[\beta(W(s))+2 \int_{0}^{s} \zeta_{1}(s) \zeta_{2}(\tau) \beta(W(\tau)) \mathrm{d} \tau\right]
\end{aligned}
$$


for a.e. $s \in[0, b]$. Then from (3.7), $\beta(W(s))=0$, we have

$$
\beta\left(\left\{f\left(s, u_{n}(s), \int_{0}^{s} h\left(s, \tau, u_{n}(\tau)\right) \mathrm{d} \tau\right)\right\}_{n=1}^{\infty}\right)=0,
$$

i.e., $\left\{f\left(s, u_{n}(s), \int_{0}^{s} h\left(s, \tau, u_{n}(\tau)\right) \mathrm{d} \tau\right)\right\}_{n=1}^{\infty}$ is relatively compact in $X$ for a.a. $s \in[0, b]$. Moreover, from the fact $\left\{u_{n}\right\}_{n=1}^{\infty} \subset W_{0}$ and hypotheses $\left(\mathrm{H}_{4}\right)$ and $\left(\mathrm{H}_{5}\right)$, we have

$$
\begin{aligned}
& \left\|f\left(s, u_{n}(s), \int_{0}^{s} h\left(s, \tau, u_{n}(\tau)\right) \mathrm{d} \tau\right)\right\| \\
& \quad \leq \theta(s)\left(\left\|u_{n}(s)\right\|+\left\|\int_{0}^{s} h\left(s, \tau, u_{n}(\tau)\right) \mathrm{d} \tau\right\|\right) \leq \theta(s)\left(r+M^{*} r\right) .
\end{aligned}
$$

Then we see that the sequence of functions $\left\{f\left(s, u_{n}(s), \int_{0}^{s} h\left(s, \tau, u_{n}(\tau)\right) \mathrm{d} \tau\right)\right\}_{n=1}^{\infty}$ is uniformly integrable for a.e. $s \in[0, b]$. So $\left\{f\left(\cdot, u_{n}(\cdot), \int_{0}^{\cdot} h\left(\cdot, \tau, u_{n}(\tau)\right) \mathrm{d} \tau\right)\right\}_{n=1}^{\infty} \subset L^{1}([0, b] ; X)$ is semicompact due to Definition 2.3. By applying Lemma 2.5 , we see that the set $\left\{G\left(f_{n}\right)\right\}_{n=1}^{\infty}$ is relatively compact in $C([0, b] ; X)$, where $(G f)(t)=\int_{0}^{t} U(t, s) f\left(s, u(s), \int_{0}^{s} h(s, \tau, u(\tau)) \mathrm{d} \tau\right) \mathrm{d} s$.

From the strong continuity of $U(t, s)$ and the compactness of $g$, we see, for each $t \in[0, b]$, that the set $\left\{U(t, 0) g\left(u_{n}\right): n \geq 1\right\}$ is relatively compact in $X$. Now, for $0 \leq t<t+h \leq b$, using the semigroup property,

$$
\left\|U(t+h, 0) g\left(u_{n}\right)-U(t, 0) g\left(u_{n}\right)\right\| \leq M\left\|[U(t+h, t)-I] g\left(u_{n}\right)\right\| .
$$

Thus the functions in $\left\{U(\cdot, 0) g\left(u_{n}\right): n \geq 1\right\}$ are equicontinuous due to the compactness of $g$ and the strong continuity of $U(t, s)$. According to the Ascoli-Arzela theorem, we get $\left\{U(\cdot, 0) g\left(u_{n}\right): n \geq 1\right\}$ is relatively compact in $C([0, b] ; X)$. So the set $\left\{K_{1} u_{n}\right\}_{n=1}^{\infty}$ is relatively compact in $C([0, b] ; X)$, noticing that $K_{1} u_{n}=G u_{n}+U(\cdot, 0) g\left(u_{n}\right)$.

Next, the same idea can be used to prove the equicontinuity of $\left\{K_{2} u_{n}\right\}_{n=1}^{\infty}$ on each $J_{i}$, $i=1,2, \ldots, p$. In fact, for $t_{i}<t<t+h \leq t_{i+1}, i=1,2, \ldots, p$, by using the semigroup properties, we have

$$
\begin{aligned}
& \left\|\left(K_{2} u_{n}\right)(t+h)-\left(K_{2} u_{n}\right)(t)\right\| \\
& \leq\left\|\sum_{0<t_{j}<t+h} U\left(t+h, t_{j}\right) I_{j}\left(u_{n}\left(t_{j}\right)\right)-\sum_{0<t_{j}<t} U\left(t+h, t_{j}\right) I_{j}\left(u_{n}\left(t_{j}\right)\right)\right\| \\
& \quad+\left\|\sum_{0<t_{j}<t} U\left(t+h, t_{j}\right) I_{j}\left(u_{n}\left(t_{j}\right)\right)-\sum_{0<t_{j}<t} U\left(t, t_{j}\right) I_{j}\left(u_{n}\left(t_{j}\right)\right)\right\| \\
& \leq \sum_{0<t_{j}<t}\left\|U\left(t+h, t_{j}\right) I_{j}\left(u_{n}\left(t_{j}\right)\right)-U\left(t, t_{j}\right) I_{j}\left(u_{n}\left(t_{j}\right)\right)\right\| \\
& \leq \sum_{0<t_{j}<t} M\left\|[U(t+h, t)-I] I_{j}\left(u_{n}\left(t_{j}\right)\right)\right\|,
\end{aligned}
$$

from which it follows that $\left\{K_{2} u_{n}\right\}_{n=1}^{\infty}$ is equicontinuous on each $J_{i}$ due to the compactness of $I_{i}$ and the strong continuity of $U(t, s)$. Similarly, $\left\{K_{2} u_{n}\right\}_{n=1}^{\infty}$ is equicontinuous at $t_{i}^{+}, i=$ $1,2, \ldots, p$.

Since $I_{i}, i=1,2, \ldots, p$, are compact, we have

$$
\beta\left(\left\{\left(K_{2} u_{n}\right)(t)\right\}_{n=1}^{\infty}\right)=\beta\left(\left\{\sum_{0<t_{i}<t} U\left(t, t_{i}\right) I_{i}\left(u_{n}\left(t_{i}\right)\right)\right\}_{n=1}^{\infty}\right)=0
$$


for $t \in[0, b]$. Then, by Lemma 2.7, we get

$$
\beta\left(\left\{K_{2} u_{n}\right\}_{n=1}^{\infty}\right)=\sup _{t \in[0, b]} \beta\left(\left\{\left(K_{2} u_{n}\right)(t)\right\}_{n=1}^{\infty}\right)=0 .
$$

From (3.9), we have

$$
\beta\left(\left\{y_{n}\right\}_{n=1}^{\infty}\right) \leq \beta\left(\left\{K_{1} u_{n}\right\}_{n=1}^{\infty}\right)+\beta\left(\left\{K_{2} u_{n}\right\}_{n=1}^{\infty}\right)
$$

which implies $\beta\left(\left\{y_{n}\right\}_{n=1}^{\infty}\right)=0$. Due to (3.8), we have

$$
\beta(K W) \leq 2 \beta\left(\left\{y_{n}\right\}_{n=1}^{\infty}\right)+\varepsilon
$$

As $\varepsilon$ is arbitrary, it follows from the above inequality that $\beta(K W)=0$. Thus, we get

$$
\beta(W)=\beta\left(\overline{\mathrm{co}}\left\{K W, u_{0}\right\}\right)=\beta(K W)=0 .
$$

Therefore, $W$ is convex compact and nonempty in $P C([0, b] ; X)$, and $K(W) \subset W$. By the Schauder fixed point theorem, there exists at least one mild solution $u$ of the problem (1.1), where $u \in W$ is a fixed point of the continuous map $K$. This completes the proof of Theorem 3.1.

Remark 3.2 If the function $f$ is compact or Lipschitz continuous (see, e.g., $[10,16])$, then $l(t)$ in hypothesis $\left(\mathrm{H}_{4}\right)$ becomes zero or a Lipschitz constant. In our proof, the measure of noncompactness (MNC) is used to get rid of the compactness of the evolution system. Note that in $[6,9,19,20], \mathrm{MNC}$ is adopted to discuss the differential and integrodifferential system in Banach spaces when the operation semigroup (evolution system) is compact or equicontinuous. Here the condition on the evolution system $U(t, s)$ is only supposed to be strongly continuous and they are weak in essence compared with the previous results. In our recent paper [27], we get some existence results of fractional differential equations with nonlocal conditions when the semigroup is strongly continuous. There the work is based on a new regular measure of noncompactness defined by us (see Lemma 3.1 of [27]). We conjecture that the two different approaches in [27] and in the present paper may be considered from a unified point of view in some way. It is an interesting problem and is worth discussing later.

\section{Applications}

Example 4.1 Consider the following integrodifferential evolution system with impulsive condition:

$$
\left\{\begin{array}{l}
\omega_{t}(t, \theta)=\omega_{\theta}(t, \theta)+F\left(t, \omega(t, \theta), \int_{0}^{t} h_{1}(t, s, \omega(s, \theta)) \mathrm{d} s\right), \quad 0 \leq t \leq b, 0 \leq \theta \leq \pi, t \neq t_{i}, \\
\omega(t, 0)=\omega(t, \pi)=0, \quad 0 \leq t \leq b, \\
\omega\left(t_{i}^{+}, \theta\right)-\omega\left(t_{i}^{-}, \theta\right)=I_{i}\left(\omega\left(t_{i}, \theta\right)\right), \quad i=1,2, \ldots, p, \\
\omega(0, \theta)=\int_{0}^{b} g_{1}(s) \log (1+|\omega(s, \theta)|) \mathrm{d} s+u_{0}(\theta), \quad 0 \leq \theta \leq \pi
\end{array}\right.
$$

where $F:[0, b] \times \mathbb{R} \times \mathbb{R} \rightarrow \mathbb{R}, h:[0, b] \times[0, b] \times \mathbb{R} \rightarrow \mathbb{R}$, and $u_{0} \in L^{2}[0, \pi]$.

Take $X=L^{2}[0, \pi]$. Define $A(t) \equiv A: D(A) \subset X \rightarrow X$ by $A z=z^{\prime}$ with the domain $D(A)=$ $\left\{z \in X: z^{\prime} \in X, z(0)=z(\pi)=0\right\}$. It is well known that $A$ is an infinitesimal generator of a 
semigroup $T(t)$ defined by $T(t) z(s)=z(t+s)$ for each $z \in X$. T(t) is not a compact semigroup on $X$ and $\beta(T(t) D) \leq \beta(D)$ for a bounded subset $D$, where $\beta$ is the Hausdorff MNC. Now, we assume that:

(1) $f:[0, b] \times X \times X \rightarrow X$ is a continuous function defined by

$$
\begin{aligned}
& f(t, x, h)(\theta)=F(t, x(t, \theta), h(t, \theta)), \quad t \in[0, b], 0 \leq \theta \leq \pi, \\
& h(t, \theta)=\int_{0}^{t} h_{1}(t, s, x(s, \theta)) \mathrm{d} s .
\end{aligned}
$$

We take

$$
F\left(t, x(t, \theta), \int_{0}^{t} h_{1}(t, s, x(s, \theta)) \mathrm{d} s\right)=c \sin (t, x(t, \theta))+c \int_{0}^{t} \sqrt{x^{2}(\xi, \theta)+1} \mathrm{~d} \xi
$$

$c$ is a constant. Then $f, h$ satisfy hypotheses $\left(\mathrm{H}_{4}\right)$ and $\left(\mathrm{H}_{5}\right)$ of Section 3.

(2) $I_{i}: X \rightarrow X$ is a continuous function for each $i=1,2, \ldots, p$, defined by

$$
I_{i}(x)(\theta)=I_{i}(x(\theta))
$$

We take

$$
I_{i}(x(\theta))=\int_{0}^{\pi} \rho_{i}(\theta, y) \cos ^{2}(x(y)) \mathrm{d} y
$$

$x \in X, \rho_{i} \in C([0, \pi] \times[0, \pi], \mathbb{R})$, for each $i=1,2, \ldots, p$. Then $I_{i}$ is compact and satisfies hypothesis $\left(\mathrm{H}_{3}\right)$.

(3) $g: P C([0, b] ; X) \rightarrow X$ is a continuous function defined by

$$
g(u)(\theta)=\int_{0}^{b} g_{1}(s) \log (1+|u(s)(\theta)|) \mathrm{d} s, \quad u \in P C([0, b] ; X)
$$

with $u(s)(\theta)=\omega(s, \theta)$. Then $g$ is a compact operator.

Under these assumptions, the above partial differential system can be reformulated as the abstract problem (1.1). Then due to Theorem 3.1, the partial differential system has at least one mild solution on $[0, b]$.

Remark 4.2 We can extend our method to exact controllability for integrodifferential evolution system with impulsive conditions. That is,

$$
\left\{\begin{array}{l}
u^{\prime}(t)=A(t) u(t)+f\left(t, u(t), \int_{0}^{t} h(t, s, u(s) \mathrm{d} s)\right)+B v(t), \\
\quad t \in J=[0, b], t \neq t_{i}, i=1,2, \ldots, p, \\
\Delta u\left(t_{i}\right)=u\left(t_{i}^{+}\right)-u\left(t_{i}^{-}\right)=I_{i}\left(u\left(t_{i}\right)\right), \quad i=1,2, \ldots, p, \\
u(0)=g(u)+u_{0},
\end{array}\right.
$$

here $B$ is a bounded linear operator from a Banach space $V$ to $X$ and the control function $v(\cdot)$ is given in $L^{2}([0, b], V)$.

Hernández and O'Regan [28] point out that some papers on exact controllability of abstract control system contain a similar technical error when the compactness of semigroup 
and other hypotheses are satisfied, that is, in this case the applications of controllability results are restricted to a finite-dimensional space. In order to fix this problem, Ji et al. [8] and Machado et al. [20] used some measures of noncompactness to weaken the assumptions of compactness on the evolution system $U(t, s)$, where the evolution system is supposed to be equicontinuous. In this paper, the evolution system is only supposed to be strongly continuous, without any restrictions of compactness or equicontinuity. Since the method used in this paper is also available for controllability of the evolution equations with impulsive conditions, we can improve many controllability results under a noncompact semigroup, like in $[8,18,20]$.

\section{Competing interests}

The authors declare that they have no competing interests.

\section{Authors' contributions}

All authors contributed equally to the writing of this paper. All authors read and approved the final manuscript.

\section{Author details}

'Faculty of Mathematics and Physics, Huaiyin Institute of Technology, Huaian, 223003, P.R. China. ${ }^{2}$ Library, Huaiyin Institute of Technology, Huaian, 223003, P.R. China.

\section{Acknowledgements}

The research is supported by Natural Science Foundation of the Jiangsu Higher Education Institutions of China (No. 14KJB1 10001), the Natural Science Foundation of Jiangsu Province (BK20150415), the Foundation of Huaiyin Institute of Technology (HGC1229).

Received: 22 April 2015 Accepted: 15 September 2015 Published online: 26 September 2015

\section{References}

1. Lakshmikantham, V, Bainov, DD, Simeonov, PS: Theory of Impulsive Differential Equations. World Scientific, Singapore (1989)

2. Benchohra, M, Henderson, J, Ntouyas, SK: Impulsive Differential Equations and Inclusions. Hindawi Publishing Corporation, New York (2006)

3. Ahmed, NU: Existence of optimal controls for a general class of impulsive systems on Banach spaces. SIAM J. Control Optim. 42, 669-685 (2003)

4. Cardinali, T, Rubbioni, P: Impulsive semilinear differential inclusion: topological structure of the solution set and solutions on non-compact domains. Nonlinear Anal. 14, 73-84 (2008)

5. Migorski, S, Ochal, A: Nonlinear impulsive evolution inclusions of second order. Dyn. Syst. Appl. 16, 155-173 (2007)

6. Fan, Z, Li, G: Existence results for semilinear differential equations with nonlocal and impulsive conditions. J. Funct. Anal. 258, 1709-1727 (2010)

7. Abada, N, Benchohra, M, Hammouche, H: Existence and controllability results for nondensely defined impulsive semilinear functional differential inclusions. J. Differ. Equ. 246, 3834-3863 (2009)

8. Ji, S, Li, G, Wang, M: Controllability of impulsive differential systems with nonlocal conditions. Appl. Math. Comput. 217, 6981-6989 (2011)

9. Ji, S, Li, G: A unified approach to nonlocal impulsive differential equations with the measure of noncompactness. Adv Differ. Equ. 2012, 182 (2012)

10. Byszewski, L: Theorems about the existence and uniqueness of solutions of a semilinear evolution nonlocal Cauchy problem. J. Math. Anal. Appl. 162, 494-505 (1991)

11. Byszewski, L, Lakshmikantham, V: Theorem about the existence and uniqueness of a solution of a nonlocal abstract Cauchy problem in a Banach space. Appl. Anal. 40,11-19 (1991)

12. Aizicovici, S, Lee, H: Nonlinear nonlocal Cauchy problems in Banach spaces. Appl. Math. Lett. 18, 401-407 (2005)

13. $\mathrm{Zhu}, \mathrm{L}, \mathrm{Li}, \mathrm{G}$ : On a nonlocal problem for semilinear differential equations with upper semicontinuous nonlinearities in general Banach spaces. J. Math. Anal. Appl. 341, 660-675 (2008)

14. Ntouyas, SK, Tsamatos, PC: Global existence for semilinear evolution equations with nonlocal conditions. J. Math. Anal. Appl. 210, 679-687 (1997)

15. Xue, X: Existence of semilinear differential equations with nonlocal initial conditions. Acta Math. Sin. 23, 983-988 (2007)

16. Xue, X: Nonlocal nonlinear differential equations with a measure of noncompactness in Banach spaces. Nonlinear Anal. TMA 70, 2593-2601 (2009)

17. Balachandran, $\mathrm{K}$, Trujillo, JJ: The nonlocal Cauchy problem for nonlinear fractional integrodifferential equations in Banach spaces. Nonlinear Anal. TMA 72, 4587-4593 (2010)

18. Arthi, G, Balachandran, K: Controllability of damped second-order impulsive neutral functional differential systems with infinite delay. J. Optim. Theory Appl. 152, 799-813 (2012)

19. Wang, J, Wei, W: A class of nonlocal impulsive problems for integrodifferential equations in Banach spaces. Results Math. 58, 379-397 (2010)

20. Machado, JA, Ravichandran, C, Rivero, M, Trujillo, J: Controllability results for impulsive mixed-type functional integro-differential evolution equations with nonlocal conditions. Fixed Point Theory Appl. 2013, 66 (2013) 
21. Benchohra, M, Ziane, M: Impulsive evolution inclusions with state-dependent delay and multivalued jumps. Electron. J. Qual. Theory Differ. Equ. 2013, 42 (2013)

22. Banas, J, Goebel, K: Measure of Noncompactness in Banach Spaces. Lect. Notes Pure Appl. Math., vol. 60. Dekker, New York (1980)

23. Pazy, A: Semigroups of Linear Operators and Applications to Partial Differential Equations. Springer, New York (1983)

24. Kamenskii, M, Obukhovskii, V, Zecca, P: Condensing Multivalued Maps and Semilinear Differential Inclusions in Banach Spaces. de Gruyter Series in Nonlinear Analysis and Applications, vol. 7. de Gruyter, Berlin (2001)

25. Cardinali, T, Rubbioni, P: On the existence of mild solutions of semilinear evolution differential inclusions. J. Math. Anal. Appl. 308, 620-635 (2005)

26. Bothe, D: Multivalued perturbation of $m$-accretive differential inclusions. Isr. J. Math. 108, 109-138 (1998)

27. Ji, S, Li, G: Solutions to nonlocal fractional differential equations using a noncompact semigroup. Electron. J. Differ. Equ. 2013, 240 (2013)

28. Hernández, E, O'Regan, D: Controllability of Volterra-Fredholm type systems in Banach spaces. J. Franklin Inst. 346, 95-101 (2009)

\section{Submit your manuscript to a SpringerOpen ${ }^{\circ}$ journal and benefit from:}

- Convenient online submission

Rigorous peer review

- Immediate publication on acceptance

- Open access: articles freely available online

- High visibility within the field

- Retaining the copyright to your article 